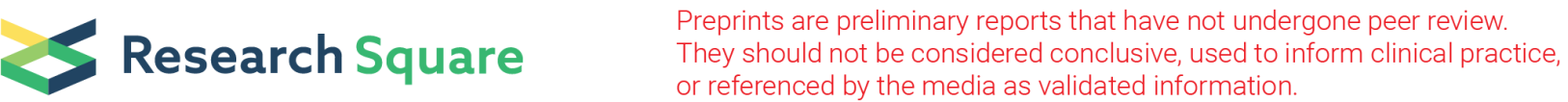

\section{Jian-Gan-Xiao-Zhi Decoction Ameliorates \\ Nonalcoholic Fatty Liver Disease Through \\ Modulating Gut Microbiota, Decreasing Gut \\ Permeability, and Alleviating Liver Inflammation}

Jiabao Liao

Jiaxing Hospital of Traditional Chinese Medicine

Xuehua Xie

Yunnan Provincial Hospital of Chinese Medicine

Jinmei Gao

Fujian People's Hospital of Traditional Chinese Medicine

Zhaiyi Zhang

Tianjin University of Traditional Chinese Medicine

Fei Qv

Jiaxing Hospital of Traditional Chinese Medicine

Huantian Cui

Shandong University

Yongjun Cao

Nantong Hospital of Traditional Chinese Medicine

Xue Han

Yunnan Provincial Hospital of Chinese Medicine

Jie Zhao

Yunnan Provincial Hospital of Chinese Medicine

Weibo Wen

Yunnan Provincial Hospital of Chinese Medicine

Hongwu Wang ( $\nabla$ whw2009@tjutcm.edu.cn )

Tianjin University of Traditional Chinese Medicine

\section{Research}

Keywords: Jian-Gan-Xiao-Zhi decoction, nonalcoholic fatty liver disease, gut microbiota, gut permeability liver inflammation

Posted Date: December 10th, 2020 
DOl: https://doi.org/10.21203/rs.3.rs-122886/v1

License: (c) (1) This work is licensed under a Creative Commons Attribution 4.0 International License. Read Full License 


\section{Abstract}

\section{Background}

Jian-Gan-Xiao-Zhi decoction (JGXZ), composed of Salvia miltiorrhiza Bunge, Panax notoginseng, Curcuma zedoaria, and other 9 types of herbs, has demonstrated beneficial effects on nonalcoholic fatty liver disease (NAFLD). However, the mechanisms behind JGXZ's impact on NAFLD remain unknown.

\section{Methods}

In this study, a NAFLD rat model induced by a high-fat diet (HFD) received oral treatment of JGXZ (8 or 16 $\mathrm{g}$ crude herb/kg) for 8 weeks. The therapeutic effects of JGXZ on NAFLD model rats were investigated through blood lipid levels and pathological liver changes. 16s rRNA analysis was used to study the changes in gut microbiota after JGXZ treatment. The expressions of occludin and tight junction protein 1 (ZO-1) in colon were investigated using immunostaining to study the effects of JGXZ on gut permeability. The anti-inflammatory effects of JGXZ were also studied through measuring the levels of IL-1 $\beta$, IL-6 and TNF-a in serum and liver.

Results

JGXZ treatment could decrease body weight and ameliorate dyslipidemia in NAFLD model rats. H\&E and Oil Red $O$ staining indicated that JGXZ reduced steatosis and infiltration of inflammatory cells into the liver. 16s rRNA analysis exhibited that JGXZ impacted the diversity of gut microbiota, decreasing the Firmicutes to Bacteroidetes ratio and increasing the relative abundance of probiotics, such as Alloprevotella, Lactobacillus, and Turicibacter. Gut permeability evaluation found that the expressions of ZO-1 and occludin in the colon were increased after JGXZ treatment, and inflammation analysis showed decreased levels of IL-1 $\beta, \mathrm{IL}-6$, and TNF- $\alpha$ in the serum and liver after treatment.

Conclusions

Our study illustrates that JGXZ ameliorates NAFLD through modulating gut microbiota, decreasing gut permeability, and alleviating liver inflammation.

\section{Background}

Nonalcoholic fatty liver disease (NAFLD) is a chronic disease characterized by hepatic steatosis and dyslipidemia that can lead to type 2 diabetes, atherosclerosis, hepatic fibrosis, and even hepatic carcinoma [1]. With lifestyles and diets changing for the worse in recent years, the morbidity of NAFLD is on the rise [2]. Currently, few therapeutic approaches have demonstrated a definite effect on NAFLD [3]. As such, there is a critical need to develop novel therapies to treat NAFLD.

Traditional Chinese medicine has been widely used in the treatment of NAFLD, with accumulating numbers of studies highlighting its lipid-lowering effects. For instance, researchers were able to utilize 
Jiang-Zhi-Ning to ameliorate high-fat diet (HFD) induced dyslipidemia through modulating the synthesis and translation of cholesterol and inhibiting oxidative stress [4]. Additionally, Zhixiong capsules have shown lipid-lowering effects on atherosclerosis model rats [5]. And Da-Huang-Ze-Xie decoction can improve dyslipidemia and hepatic steatosis in NAFLD rats through modulating gut microbiota and inhibiting the inflammatory response in liver [6].

Jian-Gan-Xiao-Zhi decoction (JGXZ)-composed of Salvia miltiorrhiza Bunge, Panax notoginseng, Curcuma zedoaria, hawthorn, Astragalus membranaceus, Vatica mangachapoi Blanco, Radix Paeoniae Rubra, Curcuma longa, Rhizoma Alismatis, Dendranthema morifolium, lotus leaf, and Glycyrrhiza uralensis Fisch.-has seen clinical use for the treatment of NAFLD. Furthermore, our previous study demonstrated that JGXZ could improve dylipidemia and insulin resistance in a NAFLD rat model [7]. However, the mechanisms behind JGXZ's beneficial impact on NAFLD remain elusive.

Part of the difficulty is that the mechanisms of pathogenesis for NAFLD itself remain unclear. An imbalance between energy consumption and intake, inflammatory responses in liver, and hereditary factors have all been implicated in NAFLD [8]. Recent studies have also indicated a critical role for gut microbiota in the progression of NAFLD, with the diversity of gut microbiota altered significantly in NAFLD patients $[9,10]$. Studies have also demonstrated that the dysfunction of the gut microbiota could impair the tight junction of intestinal epithelial cells and cause an increase in gut permeability [11]. Whereupon, metabolites derived from gut microbiota, such as lipopolysaccharide (LPS), could enter circulation through the impaired intestinal mucosal barrier gut and trigger inflammatory responses in the liver [12]. Modulating gut microbiota to improve gut permeability and reduce liver inflammation has been proposed as a potential method to alleviate NAFLD [13].

To elucidate the mechanism of JGXZ's amelioration of NAFLD, a NAFLD rat model was induced via HFD and orally treated with JGXZ (8 or $16 \mathrm{~g}$ crude herb/kg) for 8 weeks. The therapeutic effects of JGXZ on NAFLD model rats were investigated through blood lipid levels and pathological liver changes. 16s rRNA analysis was used to study the changes in gut microbiota post JGXZ treatment. Gut permeability was investigated via occludin and tight junction protein 1 (ZO-1) colon immunostaining. Lastly, the antiinflammatory effects of JGXZ were examined by quantification of IL-1 $\beta$, IL-6, and TNF-a in rat serum and livers.

\section{Materials And Methods}

\section{Reagents}

HFD ( $17.7 \%$ sucrose, $17.7 \%$ fructose, $19.4 \%$ protein and $40 \%$ fat) was purchased from Beijing Huafukang Bioscience Co., Ltd. (Beijing, China). Total DNA and RNA extraction kits, first-stand cDNA reverse transcription kits, polymerase chain reaction (PCR) kits, and primers were obtained from TianGen Biotechnology Co., Ltd. (Beijing, China). Rat IL-6, IL-1 $\beta$, and TNF-a ELISA kits were obtained from Shanghai BlueGene Biotech CO., Ltd. (Shanghai, China). Aspartate aminotransferase (AST), alanine aminotransferase (ALT), Triglyceride (TG), and Total cholesterol (TC) test kits were purchased from 
Nanjing Jiancheng Bioengineering Institute (Nanjing, china). The Oil Red $O$ staining kit was obtained from Solarbio Biotechnology Co., Ltd. (Beijing, China). Primary antibodies of ZO-1 (61-7300) and occludin (711500) were purchased from Invitrogen (USA).

\section{Animals}

Male Sprague-Dawley rats (180-220 g) were purchased from Beijing Huafukang Bioscience Co., Ltd. All animals were handled using experimental protocols outlined by the National Institutes of Health regulations and approved by the Ethics Committee and Use Committee of the Yunnan University of Traditional Chinese Medicine. Throughout the acclimatization and study periods all animals had access to food and water ad libitum and were maintained on a $12 \mathrm{~h} \mathrm{light/dark}$ cycle $\left(21 \pm 2{ }^{\circ} \mathrm{C}\right.$ with a relative humidity of $45 \pm 10 \%$ ).

\section{Preparation of JGXZ}

JGXZ contained: $15 \mathrm{~g}$ of Salvia miltiorrhiza Bunge, $6 \mathrm{~g}$ of Panax notoginseng, $15 \mathrm{~g}$ of Curcuma zedoaria, $20 \mathrm{~g}$ of hawthorn, $20 \mathrm{~g}$ of Astragalus membranaceus, $10 \mathrm{~g}$ of Vatica mangachapoi Blanco, $20 \mathrm{~g}$ of Radix Paeoniae Rubra, $12 \mathrm{~g}$ of Curcuma longa, $15 \mathrm{~g}$ of Rhizoma Alismatis, $15 \mathrm{~g}$ of Dendranthema morifolium, $15 \mathrm{~g}$ of lotus leaf, and $6 \mathrm{~g}$ of Glycyrrhiza uralensis Fisch. All herbs were purchased from the Department of Pharmacy of Yunnan Provincial Hospital of Traditional Chinese Medicine. The above herbs were soaked in $300 \mathrm{~mL}$ water for $30 \mathrm{~min}$ and decocted for $30 \mathrm{~min}$ to obtain a JGXZ extract. The water extract of JGXZ was then filtered and concentrated to a density of $4 \mathrm{~g}$ crude herb/mL.

\section{Animal Grouping}

After acclimatization to laboratory conditions for 1 week, 40 rats were weight-matched and randomized into four groups: control, model, JGXZ low-dose, and JGXZ high-dose groups. Rats in the control group received standard chow containing $59.4 \%$ total carbohydrate, $20 \%$ protein, and $4.8 \%$ fat. Rats in the model, JGXZ low-dose, and JGXZ high-dose groups received HFD for 12 weeks to induce NAFLD. Rats in the JGXZ low-dose and JGXZ high-dose groups received an oral gavage of JGXZ (8 or $16 \mathrm{~g}$ crude herb/kg rat weight, respectively) [7], whereas rats in the control and model groups received an oral treatment of 2 $\mathrm{mL}$ saline once per day for 12 weeks. Rats in each group were weighed every two weeks.

At the end of 12 weeks of JGXZ treatment, rat livers were removed and weighed under anesthesia. The liver index was calculated using the following formula: liver index (\%) = liver weight (g) / body weight (g) $\times 100$.

\section{Serum Biochemical Markers Assay}

After 12 weeks of JGXZ treatment, serum samples were collected for the biochemical assays. Briefly, rats were anaesthetized and blood was harvested by syringe from the aorta abdominalis. Then, blood was centrifuged at 3,000 rpm for 15 min to obtain the serum. The levels of TG, TC, ALT, and AST in serum were assayed according to the manufacturer's instructions provided by Nanjing Jiancheng Biological 
Engineering Institute (Nanjing, China) and the absorbance value was detected using a microplate reader (Varioskan Flash, Thermo Fisher, Massachusetts, USA).

\section{Histology}

After 12 weeks of JGXZ treatment, rat livers were removed, fixed in paraffin, and cut into $5 \mu \mathrm{m}$ sections. Hematoxylin and eosin (H\&E) staining was performed using standard protocols.

\section{Oil Red 0 Staining}

Rat livers were embedded in Tissue-Tek OCT compound (Sakura Finetek) for frozen block preparation. Frozen tissue sections were stained with Oil Red $O$ for lipid detection following the manufacturer's instructions. The staining of lipid drops by Oil Red $\mathrm{O}$ was quantified using Image $\mathrm{J}$ to obtain the integrated optical density (IOD). The mean optical density (MOD) was calculated based on the ratio of IOD to the sum area.

\section{Fecal 16S rRNA Sequencing}

After 12 weeks of JGXZ treatment, feces from the control, model, JGXZ low-dose, and JGXZ high-dose groups were simultaneously obtained under sterile conditions in a laminar flow hood. Total DNAs were extracted from fecal samples following the manufacturer's instructions. 16S rRNA sequencing was conducted as described previously [14].

\section{Cytokine Quantification by Enzyme-Linked Immunoassay (ELISA)}

The levels of IL-6, IL-1 $\beta$, and TNF- $\alpha$ in the serum were measured by ELISA according to the manufacturer's instructions (Shanghai BlueGene Biotech Co., Ltd. China).

\section{RNA Isolation and Real-Time Reverse Transcription Quantitative Polymerase Chain Reaction (qPCR)}

Total RNAs were isolated from livers using the RNA extraction kit and first strand cDNA were synthesized from $1 \mu \mathrm{g}$ of total RNA according to the manufacturer's instructions. qPCR was used to detect the expression of $\|6,\| / 1 b$, and Tnfa in liver as has been previously described [15]. All samples were run in triplicate and detected using a BIORAd QQ5 detection system. Actb was used as the loading control. Quantification was done using the $2^{-\triangle \Delta C T}$ method [16]. The sequences of all primers are listed in Table 1 .

\section{Immunostaining}

Rat colons were removed and fixed in paraffin and the expression of occludin and tight junction protein-1 (ZO-1) in the colon were accessed via immunostaining. The ratio of positive expressed area to sum area was analyzed and quantified using Image $\mathrm{J}$ based on the IOD.

\section{Statistics}


All data are reported as the mean \pm standard deviation (mean \pm SD) for independent experiments. Statistical differences between the experimental groups were examined by analysis of variance (ANOVA) using SPSS version 20.0. A $P$-value $<0.05$ was considered statistically significant. Curve-fitting was carried out using the graphical package GraphPad Prism5.

\section{Results}

\section{Effects of JGXZ on Body Weight Gain, Dyslipidemia, and Liver Pathology in NAFLD Model Rats}

During the 12 weeks of HFD treatment, the body weight in the model group increased significantly over the control group $(P<0.01)$. Both the JGXZ low-dose and high-dose treatment groups inhibited the body weight gain in HFD treated rats $(P<0.05$ and $P<0.01$, respectively Figure 1a). After JGXZ treatment for 12 weeks, the liver index was significantly higher in model group than the control group $(P<0.05)$, whereas the liver index was significantly decreased in the JGXZ high-dose group compared with the model group ( $P<0.05$, Figure 1b). Compared with the control group, the serum levels of ALT, AST, TG, and TC were significantly increased in the model group $(P<0.01$, respectively). Low-dose JGXZ treated rats exhibited significantly lower serum ALT and TG compared with rats in the model group $(P<0.05$, respectively). Accordingly, the serum levels of ALT, AST, TG, and TC were significantly lower in the JGXZ high-dose group compared with the model group $(P<0.01, P<0.01, P<0.01$ and $P<0.05$, respectively Table 2). H\&E staining indicated extensive steatosis of hepatocytes in the model group whereas JGXZ treatment alleviated hepatocyte steatosis in NAFLD model rats (Figure 1c). Likewise, Oil Red O staining showed increased lipid contents in the model group compared with the control group $(P<0.01$, Figure 1d, 1e), JGXZ (8 and $16 \mathrm{~g}$ crude herb/kg) treatment decreased the lipid liver contents $(P<0.01$, respectively Figure 1d, 1e).

\section{Effects of JGXZ on Gut Dysbiosis in NAFLD Model Rats}

We next examined whether JGXZ could improve the dysbiosis of gut microbiota using 16S rRNA sequencing. The Shannon index was calculated to determine the alpha diversity of gut microbiota in each group. The Shannon index was higher in the model group compared with the control $(P<0.01)$ and was lower in JGXZ high-dose group compared with the model group $(P<0.05$, Figure 2a). Venn diagram analysis indicated that there were 442 OTUs overlapped among the groups: 544 OTUs present in the control and model groups; 685 in the model and JGXZ low-dose groups; 695 in the model and JGXZ highdose groups; and 651 in JGXZ low-dose and JGXZ high-dose groups (Figure 2b). The beta diversity of gut microbiota was also studied using principle coordinate analysis (PCoA) and system clustering tree. PCoA and system clustering tree indicated a significant variance of beta diversity between the control and model groups. Both low-dose and high-dose JGXZ treatment changed the beta diversity in NAFLD model rats, with the distances between JGXZ treated groups (both 8 and $16 \mathrm{~g}$ crude herb/ $\mathrm{kg}$ ) and the model group in PCA shorter than that between the model and control groups. Additionally, the distance between the high-dose JGXZ treated rats and the control group showed more similar beta diversities to the control group that low-dose JGXZ treated rats (Figure 2c, 2d). 
We then investigated the changes in abundances of gut microbiota in each group. At the phylum level, Firmicutes and Bacteroidetes were the most abundant phyla in all samples (Figure 3a). The Firmicutes to Bacteroidetes ( $F$ to $B$ ) ratio was higher in the model group than in the control group $(P<0.01)$, whereas the $F$ to $B$ ratio was lower in JGXZ treated groups (8 and $16 \mathrm{~g}$ crude herb/ $\mathrm{kg}$ ) than that in the model group $(P<0.05$ and $P<0.01$, respectively Figure 3b). At the genus level, the abundances of Lactobacillus $(P<$ $0.01)$ and Blautia $(P<0.05)$ were decreased and the abundances of Turicibacter $(P<0.01)$, Collinsella $(P$ $<0.01)$, Faecalibaculum, $(P<0.05)$, and Roseburia $(P<0.01)$ were increased in model group compared with the control group (Figure 3c). Low-dose JGXZ treatment increased the abundance of Lactobacillus $(P<0.05)$ and decreased the abundances of Collinsella $(P<0.05)$ and Roseburia $(P<0.05)$. High-dose JGXZ treatment increased the abundances of Lactobacillus $(P<0.01)$ and Blautia, $(P<0.01)$ and decreased the abundances of Turicibacter $(P<0.05)$, Collinsella $(P<0.01)$, and Roseburia $(P<0.05)$ in the gut (Figure 3c).

\section{Effects of JGXZ on Gut Permeability and Liver Inflammation in NAFLD Model Rats}

Immunostaining indicated that ZO-1 and occludin were expressed in all colon epithelial cells. The expression of ZO-1 and occludin were decreased in the model group compared with the control $(P<0.01$, respectively Figure 4a-4d). JGXZ treatment ( 8 and $16 \mathrm{~g}$ crude herb/kg) increased the expression of ZO-1 $(P<0.05$ and $P<0.01$, respectively Figure $4 \mathrm{a}, 4 \mathrm{c})$ and occludin $(P<0.01$, respectively Figure $4 \mathrm{~b}, 4 \mathrm{~d})$ in HFD treated rats. The serum levels of IL-6, IL-1 $\beta$, and TNF-a were increased in NAFLD model rats compared with rats receiving standard chow $(P<0.01$, respectively Figure $4 \mathbf{e})$. The serum levels of IL-1 $\beta$ and TNF-a were lower in the JGXZ low-dose group than those in the model group $(P<0.05$ and $P<0.01$, respectively Figure 4e). High-dose JGXZ treatment decreased the serum levels of IL-6, IL-1 $\beta$, and TNF-a in NAFLD model rats $(P<0.01$, respectively Figure $4 \mathrm{e})$. Likewise, the gene expression of $\|6\| ,1 \mathrm{~b}$, and Tnfa in liver were upregulated in the model group compared with the control group $(P<0.01$, respectively). Whereas, JGXZ treatment (8 and $16 \mathrm{~g}$ crude herb/kg) downregulated the expression of $/ 16(P<0.05$ and $P$ $<0.01$, respectively), $/ / 1 b,(P<0.01$, respectively) and $\operatorname{Tnfa}(P<0.01$, respectively) in the liver (Figure 4f).

\section{Discussion}

In this study, we established a NAFLD rat model using HFD. Our results showed that the body weights and liver indices were increased in rats receiving a HFD. Moreover, NAFLD model rats exhibited significant body weight gain, dyslipidemia, and hepatic steatosis which are consistent with the pathological changes of NAFLD. In agreement with our previous study, JGXZ treatment ( 8 and $16 \mathrm{~g}$ crude herb/ $\mathrm{kg}$ ) showed remarkable therapeutic effects on NAFLD, manifesting as an improvement of body weight gain, liver index, dyslipidemia, and pathological liver changes.

In addition, we investigated changes in gut microbiological composition using high-throughput sequencing. HFD has been shown previously to cause an increase in the alpha diversity of gut microbiota [17]. Likewise, our results showed higher Shannon indexes in HFD treated rats compared with the control group. JGXZ (16 g crude herb/ $\mathrm{kg}$ ) treatment reduced the gut microbiota alpha diversity of NAFLD rats. 
PCOA analysis revealed significant distances between control and NAFLD model rats, indicating that the beta diversity of gut microbiota differed in HFD rats from rats receiving standard chow. According to the system clustering tree, the beta diversity of the gut microbiota between JGXZ (16 g crude herb/ $\mathrm{kg}$ ) treated rats and control rats were more alike than the NAFLD model rats to control rats. Accumulating numbers studies show that the ratio between Firmicutes to Bacteroidetes ( $\mathrm{F}$ to $\mathrm{B}$ ratio) is closely related to many metabolic diseases, including obesity, type 2 diabetes, and NAFLD [18-20]. Compared with the healthy subjects, NAFLD patients show a significant increase in the relative abundance of Firmicutes and a remarkable decrease in Bacteroidetes, resulting in an increase of $F$ to $B$ ratio [21-22]. Decreasing the $F$ to $B$ ratio in the gut microbiota demonstrated a superior clinical outcome in NAFLD patients [23]. In agreement these prior studies, our results indicated a remarkable increase of $F$ to $B$ ratio in the model group, with JGXZ treatment decreasing this increased $\mathrm{F}$ to $\mathrm{B}$ ratio.

At the genus level, the abundances of Lactobacillus and Blautia were increased in JGXZ treated rats. Previous studies have demonstrated that Lactobacillus and Blautia were decreased in NAFLD patients [24-25]. Mulberry leaf fiber and polyphenols mixture have been used to induce weight loss in obese rats through increasing the abundance of Lactobacillus [26]. Similarly, Xie-Xin-Tang has been shown to increase the abundance of Blautia and improve the hyperglycemia, dyslipidemia, and inflammation in type 2 diabetes rats [27].

The relative abundances of Turicibacter, Collinsella, Faecalibaculum, and Roseburia were all increased in rats fed with HFD; JGXZ treatment decreased the gut abundances of Turicibacter, Collinsella, and Roseburia. Studies have shown that Turicibacter is positively correlated with metabolic phenotypes induced by HFD [28]. Ethanol extracts from marine microalga Chlorella pyrenoidosa have been shown to alleviate lipid metabolic disorders in HFD rats through decreasing the abundance of Turicibacter [29]. In addition, clinical studies have demonstrated that the abundance of Collinsella was significantly increased in atherosclerotic and NASH patients [30]. Additional correlation analysis indicated that Collinsella was positively related to the dysfunction of lipid metabolism in nonalcoholic steatohepatitis (NASH) patients [31]. Furthermore, Collinsella was decreased in obese type 2 diabetic patients after they received a structured weight loss program [32]. The effects of JGXZ on dyslipidemia have been previously attributed to decreases of Turicibacter and Collinsella in gut [33].

Changes in dietary habits could be responsible for changes in Faecalibaculum abundance. For example, one study found that the abundance of Faecalibaculum was increased in mice receiving whole-egg powder vs a normal diet [34]. As for the changes in Rosenburia, the abundance of Roseburia has been shown to increase in aged mice. However, few studies have demonstrated a role for Faecalibaculum or Roseburia in NAFLD and further study is required before conclusions on their importance can be drawn [35].

As the dysfunction in gut microbiota can disrupt the tight junction of intestinal epithelial cells, triggering an increase in gut permeability and contributing to liver inflammation [36], we studied whether JGXZ could enhance the integrity of the intestinal mucosa barrier and ameliorate inflammation in NAFLD model 
rats. ZO-1 and occludin were examined as indicators as they are tight junction proteins expressed in the intestinal epithelial cells [37]. Occludin can influence the intestinal epithelial tight junction barrier through regulating macromolecule flux [38]. Z0-1, also called tight junction protein 1, is a cytoplasmic plaque

protein that connects the transmembrane proteins to cytoskeleton [39,40]. Decreases in ZO-1 and occludin expression in the gut indicate a reduction of intestinal epithelial cell integrity [41]. Accordingly, reduced levels of ZO-1 and occludin were observed in HFD treated rats compared with control rats, whereas, JGXZ treatment increased the protein expression of both ZO-1 and occludin. Moreover, JGXZ treatment attenuated the inflammatory response in NAFLD model rats, manifesting as mild lobular liver inflammation and reduced expression of pro-inflammatory cytokines (IL-6, IL-1 $\beta$, and TNF-a) in the serum and liver. Infiltration of inflammatory cells into the liver can induce excessive pro-inflammatory cytokine production, including IL-6, IL-1 $\beta$, and TNF-a, and induce hepatocyte injury, contributing to NASH [42]. These pro-inflammatory cytokines can also disrupt lipid metabolism and cause dyslipidemia [43]. Previous studies have demonstrated that the colonization of Lactobacillus rhamnosus GG can prevent liver injury by improving the gut integrity and ameliorating liver inflammation in an alcoholic liver disease model [44]. Lactobacillus rhamnosus GG was also shown to improve intestinal barrier dysfunction in patients with irritable bowel syndrome [45]. Blautia has been reported to reduce the inflammatory response in obesity-related complications. In vitro studies have shown that Blautia can inhibit the production of pro-inflammatory cytokines in the LPS-induced activation of peripheral blood mononuclear cells [46]. Based on these previous researches, we postulate that the effects of JGXZ on gut permeability and liver inflammation occur via affecting the abundances of Lactobacillus and Blautia [47].

\section{Conclusion}

In conclusion, our current study demonstrated that JGXZ could ameliorate NAFLD through modulating gut microbiota, decreasing gut permeability, and alleviating liver inflammation (Figure 5). Rats receiving high-dose JGXZ treatment exhibited superior therapeutic outcomes-more significant improvement in gut microbiota dysbiosis, lower grade of inflammation, and higher gut permeability -than low-dose JGXZ treated rats, highlighting JGXZ's mechanisms and therapeutic potential against NAFLD in a dosedependent manner.

\section{Declarations}

\section{Ethics approval and consent to participate}

All animals were handled using experimental protocols outlined by the National Institutes of Health regulations and approved by the Ethics Committee and Use Committee of the Yunnan University of Traditional Chinese Medicine.

\section{Acknowledgements}

Not applicable. 


\section{Authors' Contributions}

L.-J.B., X.-X.H., and G.-J.M. conceived and designed the research methods. L.-J.B., X.-X.H., G.-J.M., Z.-Z.Y., Q. F., C.-H.T., J.Z., W.-H.W., and W.-W.B. collected the data. W.-H.W., and W.-W.B. analyzed the data.

G.-J.M., Z.-Z.Y., and W.-H.W., wrote the original draft. L.-J.B., and X.-X.H. reviewed and edited the manuscript. All authors have read and agreed to the published version of the manuscript.

\section{Funding}

This work was funded by the National Science Foundation of China (81560772)

\section{Availability of data and materials}

The datasets used and/or analysed during the current study are available from the corresponding author on reasonable request.

\section{Consent for publication}

Not applicable.

\section{Competing interests}

The authors declare no conflict of interest.

\section{References}

1 Dibba P, Li A, Cholankeril G, Iqbal U, Gadiparthi C, Khan MA, et al. Mechanistic Potential and Therapeutic Implications of Cannabinoids in Nonalcoholic Fatty Liver Disease. Medicines (Basel). 2018; 5(2): 47.

2 Zelber-Sagi S, Godos J, Salomone F. Lifestyle changes for the treatment of nonalcoholic fatty liver disease: a review of observational studies and intervention trials. Therap Adv Gastroenterol. 2016; 9(3): 392-407.

3 Rosato V, Abenavoli L, Federico A, Masarone M, Persico M. Pharmacotherapy of alcoholic liver disease in clinical practice. Int J Clin Pract. 2016;70(2):119-131.

4 Chen J, Zhao H, Yang Y, Liu B, Ni J, Wang W. Lipid-lowering and antioxidant activities of Jiang-Zhi-Ning in Traditional Chinese Medicine. J Ethnopharmacol. 2011; 134(3): 919-30.

5 Zhai J, Ren Z, Wang Y, Han M, Han N, Liu Z, et al. Traditional Chinese patent medicine Zhixiong Capsule (ZXC) alleviated formed atherosclerotic plaque in rat thoracic artery and the mechanism investigation including blood-dissolved-component-based network pharmacology analysis and biochemical validation. J Ethnopharmacol. 2020; 254: 112523. 
6 Fang J, Sun X, Xue B, Fang N, Zhou M. Dahuang Zexie Decoction Protects against High-Fat DietInduced NAFLD by Modulating Gut Microbiota-Mediated Toll-Like Receptor 4 Signaling Activation and Loss of Intestinal Barrier. Evid Based Complement Alternat Med. 2017; 2017: 2945803.

7 Xie XH, Liao JB, Fang F, Zhao J, Cao YJ, Cui HT, et al. Jian-Gan-Xiao-Zhi decoction ameliorates high-fat high-carbohydrate diet-induced non-alcoholic fatty liver disease and insulin resistance by regulating the AMPK/JNK pathway. Traditional Medicine Research 2021, 6 (1): 4.

8 Ekhlasi G, Zarrati M, Agah S, Hosseini AF, Hosseini S, Shidfar S, er al. Effects of symbiotic and vitamin E supplementation on blood pressure, nitric oxide and inflammatory factors in non-alcoholic fatty liver disease. EXCLI J. 2017; 16: 278-290.

9 Leung C, Rivera L, Furness JB, Angus PW. The role of the gut microbiota in NAFLD. Nat Rev Gastroenterol Hepatol. 2016; 13(7): 412-25.

10 Del Chierico F, Nobili V, Vernocchi P, Russo A, De Stefanis C, Gnani D, et al. Gut microbiota profiling of pediatric nonalcoholic fatty liver disease and obese patients unveiled by an integrated meta-omics-based approach. Hepatology. 2017; 65(2): 451-464.

11 Lin Y, Li B, Yang X, Liu T, Shi T, Deng B, et al. Non-hematopoietic STAT6 induces epithelial tight junction dysfunction and promotes intestinal inflammation and tumorigenesis. Mucosal Immunol. 2019;12(6):1304-1315.

12 Bhat MI, Kapila R. Dietary metabolites derived from gut microbiota: critical modulators of epigenetic changes in mammals. Nutr Rev. 2017;75(5):374-389.

13 Ding Y, Yanagi K, Cheng C, Alaniz RC, Lee K, Jayaraman A. Interactions between gut microbiota and non-alcoholic liver disease: The role of microbiota-derived metabolites. Pharmacol Res. 2019; 141: 521529.

14 Li JC, Cui HT, Cai YZ, Lin J, Song X, Zhou ZJ, et al. Tong-Xie-Yao-Fang Regulates 5-HT Level in Diarrhea Predominant Irritable Bowel Syndrome Through Gut Microbiota Modulation. Front Pharmacol. 2018; 9:1110.

15 Cui HT, Cai YZ, Wang L, et al. Berberine Regulates Treg/Th17 Balance to Treat Ulcerative Colitis Through Modulating the Gut Microbiota in the Colon. Front Pharmacol. 2018; 9: 571.

16 Livak KJ, Schmittgen TD. Analysis of relative gene expression data using real-time quantitative PCR and the 2(-Delta Delta C(T)) Method. Methods. 2001;25(4):402-8.

17 Lecomte V, Kaakoush NO, Maloney CA, Raipuria M, Huinao KD, Mitchell HM, et al. Changes in gut microbiota in rats fed a high fat diet correlate with obesity-associated metabolic parameters. PLoS One. 2015; 10(5): e0126931. 
18 Indiani CMDSP, Rizzardi KF, Castelo PM, Ferraz LFC, Darrieux M, Parisotto TM. Childhood Obesity and Firmicutes/Bacteroidetes Ratio in the Gut Microbiota: A Systematic Review. Child Obes. 2018; 14(8): 501509.

19 Magne F, Gotteland M, Gauthier L, Zazueta A, Pesoa S, Navarrete P, et al. The Firmicutes/Bacteroidetes Ratio: A Relevant Marker of Gut Dysbiosis in Obese Patients? Nutrients. 2020;12(5):1474.

20 Monga Kravetz A, Testerman T, Galuppo B, Graf J, Pierpont B, Siebel S, et al. Effect of Gut Microbiota and PNPLA3 rs738409 Variant on Nonalcoholic Fatty Liver Disease (NAFLD) in Obese Youth. J Clin Endocrinol Metab. 2020;105(10): dgaa382.

21 Pataky Z, Genton L, Spahr L, Lazarevic V, Terraz S, Gaïa N, et al. Impact of Hypocaloric Hyperproteic Diet on Gut Microbiota in Overweight or Obese Patients with Nonalcoholic Fatty Liver Disease: A Pilot Study. Dig Dis Sci. 2016;61(9):2721-31.

22 Chang CC, Lin KY, Peng KY, Day YJ, Hung LM. Resveratrol exerts anti-obesity effects in high-fat diet obese mice and displays differential dosage effects on cytotoxicity, differentiation, and lipolysis in 3T3L1 cells. Endocrine Journal. 2016; 63(2):169-178.

23 Bell, David SH. Changes seen in gut bacteria content and distribution with obesity: causation or association? Postgraduate Medicine. 2015; 127(8):1-6.

24 Vajro P, Paolella G, Fasano A. Microbiota and gut-liver axis: their influences on obesity and obesityrelated liver disease. J Pediatr Gastroenterol Nutr. 2013; 56(5): 461-8.

25 Fukui H. Gut Microbiota and Host Reaction in Liver Diseases. Microorganisms. 2015; 3(4): 759-91.

26 Li Q, Liu F, Liu J, Liao S, Zou Y. Mulberry Leaf Polyphenols and Fiber Induce Synergistic Antiobesity and Display a Modulation Effect on Gut Microbiota and Metabolites. Nutrients. 2019;11(5):1017.

27 Wei X, Tao J, Xiao S, Jiang S, Shang E, Zhu Z, et al. Xiexin Tang improves the symptom of type 2 diabetic rats by modulation of the gut microbiota. Sci Rep. 2018;8(1):3685.

28 Zhong Y, Nyman M, Fåk F. Modulation of gut microbiota in rats fed high-fat diets by processing wholegrain barley to barley malt. Mol Nutr Food Res. 2015;59(10):2066-76.

29 Wan X, Li T, Liu D, Chen Y, Liu Y, Liu B, et al. Effect of Marine Microalga Chlorella pyrenoidosa Ethanol Extract on Lipid Metabolism and Gut Microbiota Composition in High-Fat Diet-Fed Rats. Mar Drugs. 2018;16(12):498.

30 Karlsson FH, Fåk F, Nookaew I, Tremaroli V, Fagerberg B, Petranovic D, et al. Symptomatic atherosclerosis is associated with an altered gut metagenome. Nat Commun. 2012; 3: 1245. 
31 Astbury S, Atallah E, Vijay A, et al. Lower gut microbiome diversity and higher abundance of proinflammatory genus Collinsella are associated with biopsy-proven nonalcoholic steatohepatitis. Gut Microbes, 2019:1-12.

32 Frost F, Storck LJ, Kacprowski T, et al. A structured weight loss program increases gut microbiota phylogenetic diversity and reduces levels of Collinsella in obese type 2 diabetics: A pilot study. PLoS ONE, 2019, 14(7): e0219489-.

33 Wan X, Li T, Liu D, et al. Effect of Marine Microalga Chlorella pyrenoidosa Ethanol Extract on Lipid Metabolism and Gut Microbiota Composition in High-Fat Diet-Fed Rats. Mar Drugs. 2018;16(12):498.

34 Fukunaga M, Suriki K, Kuda T, et al. Typical indigenous bacteria in the cecum of ddY mice fed a casein-beef tallow diet or whole-egg diet. J Food Biochem. 2019;43(12): e13064.

35 Raman M, Ahmed I, Gillevet PM, et al. Fecal Microbiome and Volatile Organic Compound Metabolome in Obese Humans With Nonalcoholic Fatty Liver Disease. Clinical Gastroenterology \& Hepatology, 2013, 11(7):868.

36 Bulló M, García-Lorda P, Megias I, et al. Systemic inflammation, adipose tissue tumor necrosis factor, and leptin expression. Obes Res. 2012; 11(4):525-531.

37 Zhang J, Wu G, Shan A, et al. Dietary glutamine supplementation enhances expression of ZO-1 and occludin and promotes intestinal development in Min piglets. Acta Agriculturae Scandinavica, Section A Animal Science. 2017:1-7.

38 Al-Sadi R, Khatib K, Guo S, Ye D, Youssef M, Ma T.Occludin regulates macromolecule flux across the intestinalepithelial tight junction barrier.AJP: Gastrointestinal and Liver Physiology. 2011; 300(6): G1054G1064.

39 Sheng M, Sala C. PDZ DOMAINS AND THE ORGANIZATION OF SUPRAMOLECULAR COMPLEXES. Annual Review of Neuroscience. 2001; 24(1):1.

40 Ranganathan R, Ross EM. PDZ domain proteins: scaffoldsfor signaling complexes. Current Biology. 1997; 7(12):0-0.

41 Han XN, Fink MP, Delude RL. Inflammatory cytokines decrease ZO-1 and occludin expression in intestinal epithelial cells by increasing no center dot production. Critical Care Medicine, 2001, 29(12): A58A58.

42 Capurso C, Capurso A. From excess adiposity to insulin resistance: The role of free fatty acids. Vascular Pharmacology. 2012; 57(2-4):91-97.

43 Liu J, Yue S, Yang Z, et al. Oral hydroxysafflor yellow A reduces obesity in mice by modulating the gut microbiota and serum metabolism. Pharmacological Research. 2018; 134. 
44 Bruch-Bertani JP, Uribe-Cruz C, Pasqualotto A, et al. Hepatoprotective Effect of Probiotic Lactobacillus rhamnosus GG Through the Modulation of Gut Permeability and Inflammasomes in a Model of Alcoholic Liver Disease in Zebrafish. Journal of the American College of Nutrition, 2019, 39(3):1-8.

$45 \mathrm{Xu} \mathrm{H}$, Allen L, Sha H, et al. Lactobacillus rhamnosus GG prevents epithelial barrier dysfunction induced by interferon-gamma and fecal supernatants from irritable bowel syndrome patients in human intestinal enteroids and colonoids. Gut Microbes, 2018:00-00.

46 Benítez-Páez A, Gómez Del Pugar EM, López-Almela I, Moya-Pérez Á, Codoñer-Franch P, Sanz Y. Depletion of Blautia Species in the Microbiota of Obese Children Relates to Intestinal Inflammation and Metabolic Phenotype Worsening. mSystems. 2020;5(2):e00857-19.

47 Benítez-Páez A, Gómez Del Pugar EM, López-Almela I, Moya-Pérez Á, Codoñer-Franch P, Sanz Y. Depletion of Blautia Species in the Microbiota of Obese Children Relates to Intestinal Inflammation and Metabolic Phenotype Worsening. mSystems. 2020; 5(2): e00857-19.

\section{Tables}

Table 1 Primer sequences used for the target rat genes

\begin{tabular}{|ll|}
\hline Genes & Primer sequence (5' $^{\prime} \mathbf{3}^{\prime}$ ) \\
\hline Actb & Forward: TCTTCCAGCCTTCCTTCCTG \\
& Reverse: CACACAGAGTACTTGCGCTC \\
\hline I/6 & $\begin{array}{ll}\text { Forward: CTCATTCTGTCTCGAGCCCA } \\
\text { Reverse: TGAAGTAGGGAAGGCAGTGG }\end{array}$ \\
\hline Tnfa & Forward: GGGATGATGACGACCTGCTA \\
& Reverse: TGTCGTTGCTTGTCTCTCCT \\
\hline
\end{tabular}

Table 2 Changes in serum ALT, AST, TG, and TC levels in NAFLD model rats 


\begin{tabular}{|lllll|}
\hline Group & ALT (U/L) & AST (U/L) & TG (mmol/L) & TC $(\mathrm{mmol} / \mathrm{L})$ \\
\hline Control & $36.87 \pm 8.61$ & $81.33 \pm 16.15$ & $2.06 \pm 0.49$ & $4.46 \pm 1.26$ \\
\hline Model & $77.02 \pm 18.80^{\# \#}$ & $166.82 \pm 41.02^{\# \#}$ & $10.67 \pm 2.07^{\# \#}$ & $6.82 \pm 1.51^{\# \#}$ \\
\hline JGXZ low-dose & $53.12 \pm 21.33^{*}$ & $145.19 \pm 38.16$ & $7.25 \pm 2.60^{*}$ & $6.05 \pm 2.08$ \\
\hline JGXZ high-dose & $45.80 \pm 9.97^{* *}$ & $129.81 \pm 17.02^{* \star}$ & $6.37 \pm 1.57^{* *}$ & $4.69 \pm 1.49^{*}$ \\
\hline
\end{tabular}

Control, model, JGXZ low-dose, and JGXZ high-dose groups ( $\mathrm{n}=10$ per group). Data are presented as the mean \pm SD. ${ }^{\# \#: ~} P<0.01$ as compared to the control group; ${ }^{*}: P<0.05$ as compared to the experimental model group; ${ }^{* *}: P<0.01$ as compared to the experimental model group.

\section{Figures}




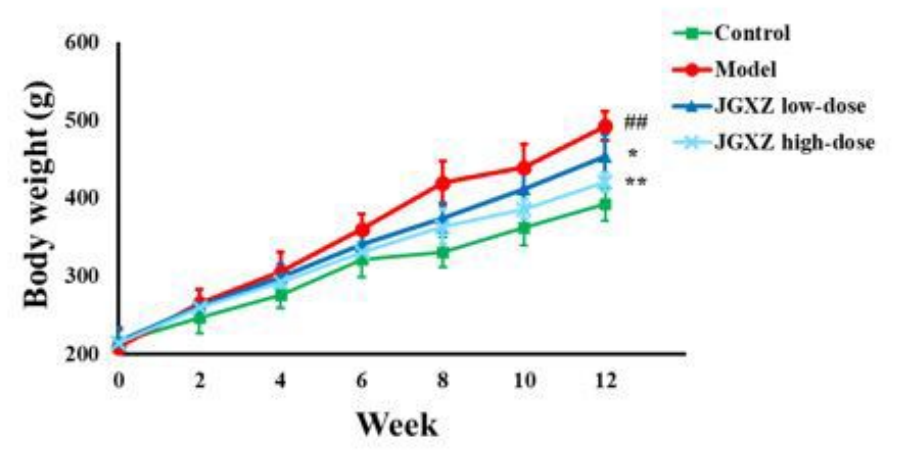

b

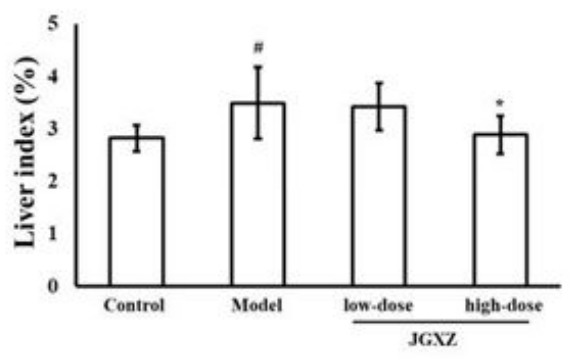

c
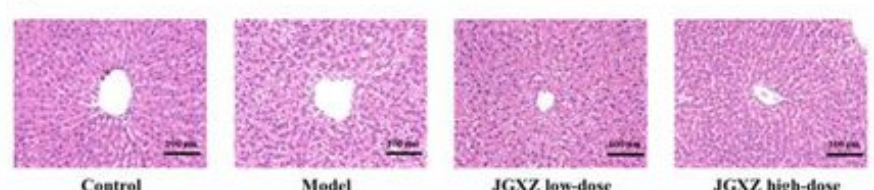

d
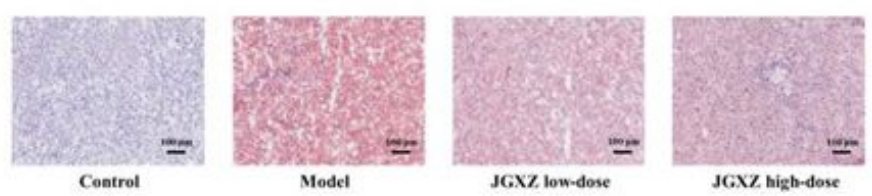

e

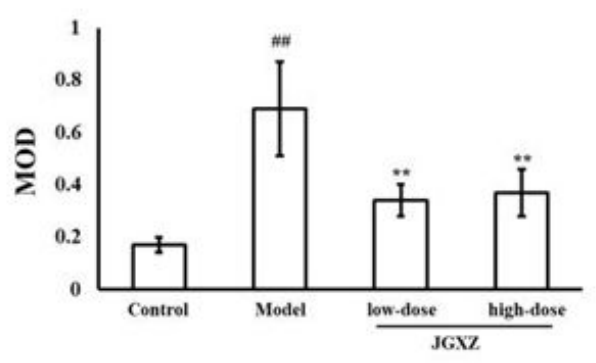

\section{Figure 1}

JGXZ treatment reduced body weight gain and improved liver steatosis in NAFLD model rats. (a) Body weight gain was reduced in NAFLD model rats after treatment with JGXZ. (b) JGXZ treatment decreased the liver index in NAFLD model rats. (c) H\&E staining indicated that JGXZ treatment ameliorated liver steatosis in NAFLD model rats (200x). (d,e) The liver lipid contents were decreased in NAFLD model rats after JGXZ treatment (100x). Control, model, JGXZ low-dose, and JGXZ high-dose groups ( $\mathrm{n}=10$ per 
group). Data are presented as the mean $\pm S D$. \#: $P<0.05$ as compared to the control group; \#\#: $P<0.01$ as compared to the control group; $*$ : $P<0.05$ as compared to the experimental model group; $* *: P<0.01$ as compared to the experimental model group.

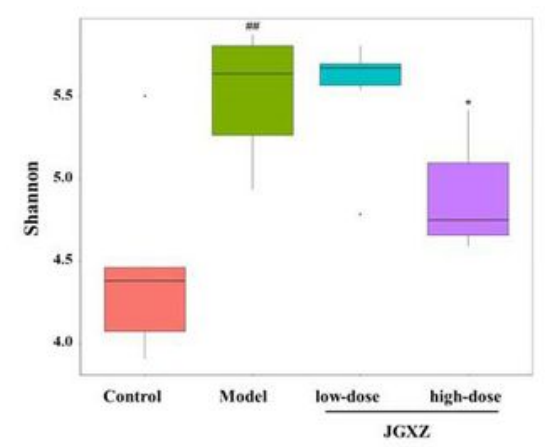

b

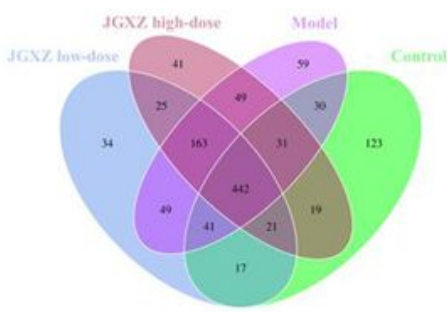

c

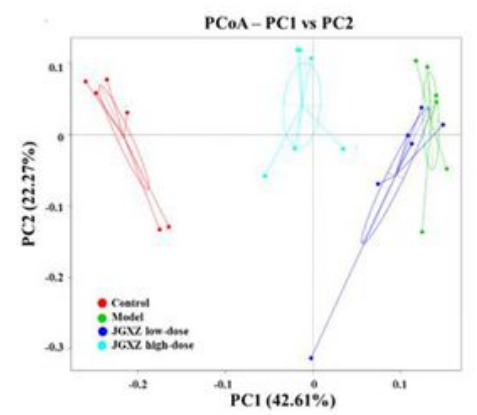

d

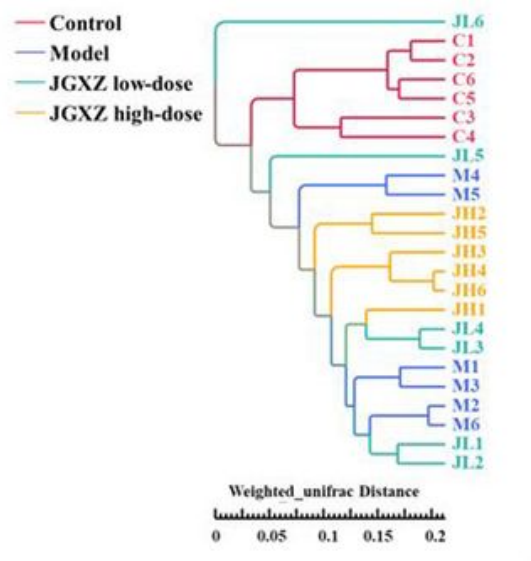

Figure 2

JGXZ treatment improved the diversity of gut microbiota in NAFLD model rats. (a) The Shannon index was decreased in NAFLD model rats after JGXZ treatment. (b) The different numbers of OTUs were 
visualized via a Venn diagram. (c, d) PCoA and system clustering tree showed more similar beta diversity between the JGXZ high-dose and control groups than that between the model and control groups.

Control, model, JGXZ low-dose, and JGXZ high-dose groups ( $n=6$ per group). \#\#: $P<0.01$ as compared to the control group; *: $\mathrm{P}<0.05$ as compared to the experimental model group.

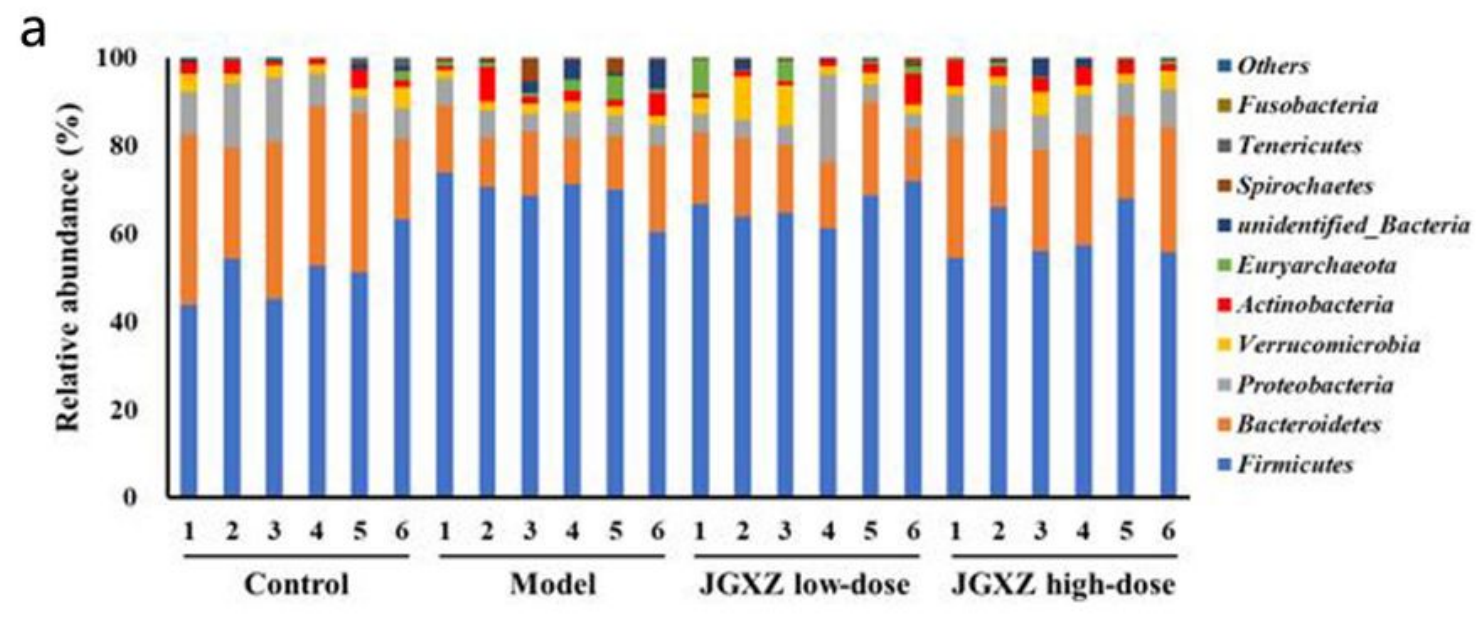

b

$F$ to $B$ ratio

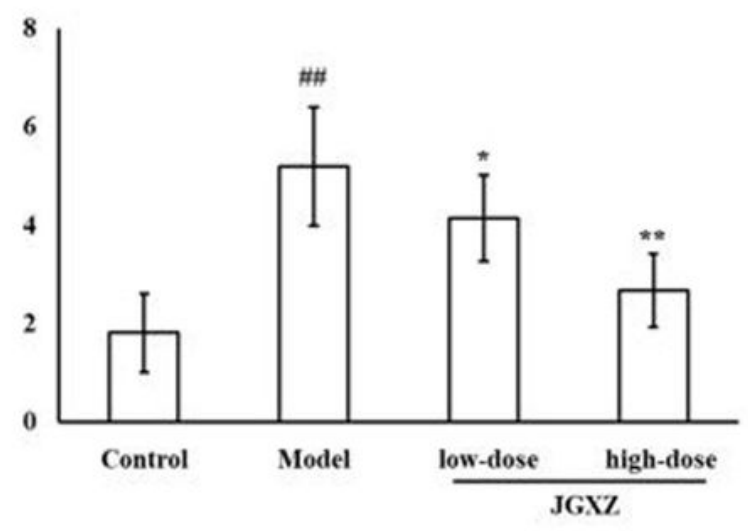

c

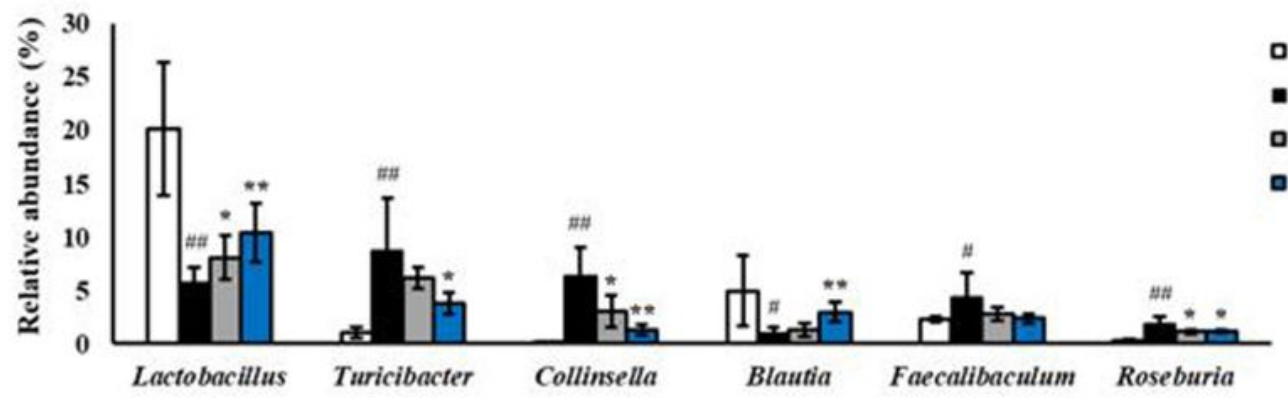

Figure 3 
JGXZ treatment altered the abundances of gut microbiota in NAFLD model rats. $(a, b)$ At the phylum level, JGXZ treatment decreased the $F$ to $B$ ratio in NAFLD model rats. (c) JGXZ treatment increased the abundances of Lactobacillus and Blautia and decreased the abundances of Turicibacter, Collinsella and Roseburia in the gut. Control, model, JGXZ low-dose, and JGXZ high-dose groups ( $\mathrm{n}=6$ per group). \#: $\mathrm{P}<$ 0.05 as compared to the control group; \#\#: $P<0.01$ as compared to the control group; $*$ : $P<0.05$ as compared to the experimental model group; $* *: P<0.01$ as compared to the experimental model group.
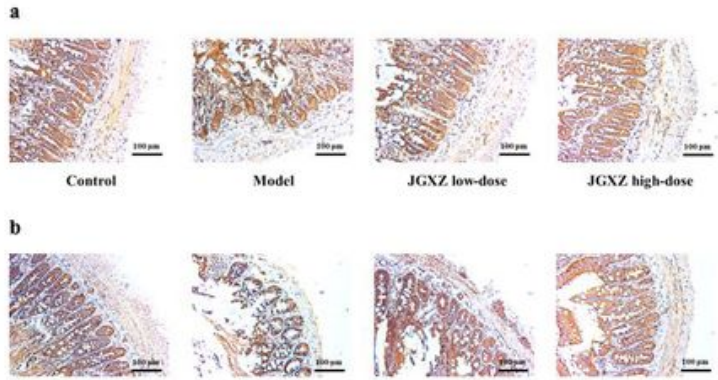

Model

JGXZ low-dose

JGXZ high-dose

Control
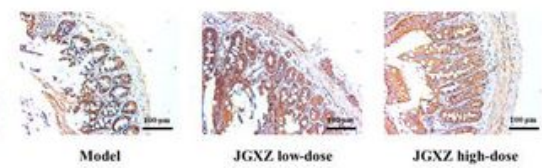

c
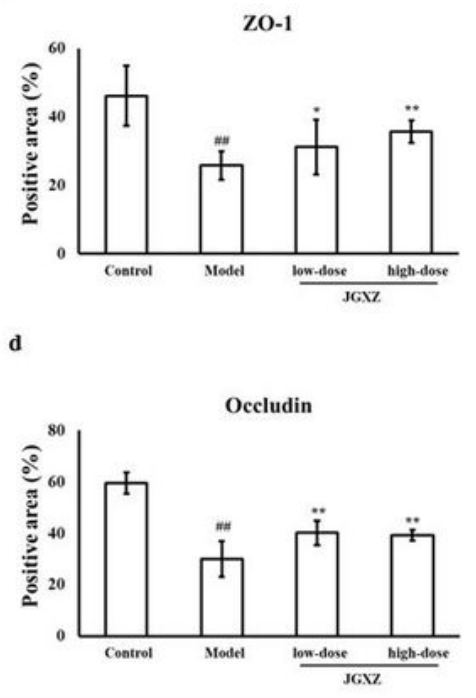

e

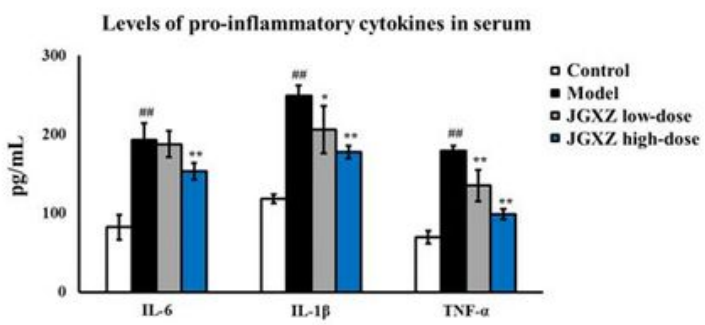

f

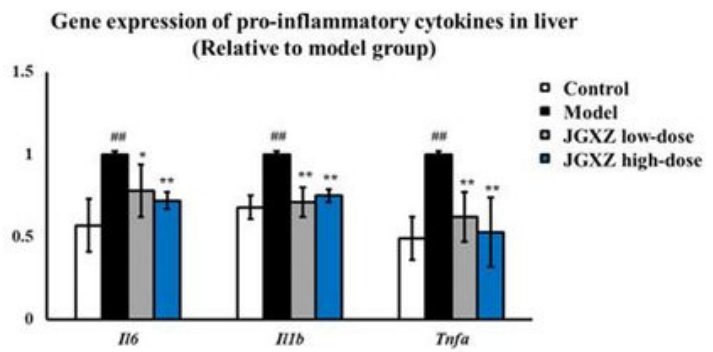

Figure 4 
JGXZ treatment increased gut integrity and inhibited the inflammatory response in NAFLD model rats. (ad) Immunostaining showed that the expression of ZO-1 (a, c) and occludin (b, d) in the colon was increased after JGXZ treatment. (e) The levels of IL-6, IL-1 $1 \beta$, and TNF- $\alpha$ in the serum were decreased after JGXZ treatment. (f) The gene expression of $\|6\| ,1 \mathrm{~b}$, and Tnfa in the liver were decreased after JGXZ treatment. Control, model, JGXZ low-dose, and JGXZ high-dose groups ( $n=10$ per group). \#: $P<0.05$ as compared to the control group; \#\#: $\mathrm{P}<0.01$ as compared to the control group; $*$ : $<0.05$ as compared to the experimental model group; $* *: P<0.01$ as compared to the experimental model group.

\section{Supplementary Files}

This is a list of supplementary files associated with this preprint. Click to download.

- abstract.JPG 